\title{
POSIBILIDADES DEL USO DE TRAMAS ARTIFICIALES DE IMAGEN MOTORA PARA UN BCI BASADO EN EEG
}

\author{
Josep Dinarès-Ferran ${ }^{1,2}$ \\ (dinares@gtec.at, josep.dinares@uvic.cat) \\ Christoph Guger ${ }^{1}$ y Jordi Solé-Casals ${ }^{2}$ \\ ${ }^{1}$ g.tec medical engineering Spain SL, C/ Plom 5, 08038 Barcelona, España \\ ${ }^{2}$ Universitat de Vic - Universitat Central de Catalunya, C/ de la Laura 13, 08500, Vic, España
}

\begin{abstract}
Resumen
La utilización de una interfaz cerebro-computadora basada en el paradigma de la imagen motora requiere un entrenamiento para adaptar el sistema al cerebro del usuario. Este proceso de adecuación para crear un clasificador útil requiere un número muy elevado de tramas de señal EEG, y adquirir esas tramas requiere tiempo. Así, ahorrar tiempo de entrenamiento puede incrementar el tiempo dedicado al uso de la aplicación de la interfaz. Utilizando la descomposición EMD (Empirical Mode Decomposition) sobre las tramas EEG y mezclando sus IMFs (Intrinsic Mode Function), es posible crear nuevas tramas EEG artificiales e incrementar el número total de tramas para entrenar el clasificador. Los resultados preliminares del estudio muestran que las tasas de error de los clasificadores creados con tramas reales y artificiales mezcladas son similares a las tasas de error del clasificador creado solo con tramas reales. Estos resultados deberán ser confirmados con estudios futuros donde se pueda hacer una validación estadística de los mismo.
\end{abstract}

Palabras Clave: BCI, EMD, Empirical Mode Decomposition, Interfaz cerebro-computadora, imagen motora, EEG.

\section{INTRODUCCIÓN}

Las interfaces cerebro-Computadora (ICC), o BrainComputer Interfaces (BCI) en inglés, son sistemas capaces de controlar dispositivos externos solo con las señales del cerebro [1]. Un BCI se divide en tres partes principales:

1. Sistema de adquisición de las señales cerebrales.

2. Sistema de procesamiento.

3. Sistema externo.

Hay diferentes sistemas de adquisición y su elección depende de la aplicación a dar al BCI [1], [2]. La utilización de gorros EEG (Electroencefalograma) permite conseguir sistemas no invasivos con una resolución temporal alta, y que son adecuados para aplicaciones en tiempo real [2]. Las señales EEG son diferencias de potencial eléctrico de distintos puntos del cuero cabelludo y son la respuesta a la activación o disparo de las neuronas como respuesta a un estímulo externo. La sincronización a esta respuesta de diferentes grupos de neuronas dará lugar a una mayor diferencia de potencial que puede ser captado y transferido al sistema de procesamiento.

Un ordenador, o una unidad de procesamiento, hará la tarea de procesar las señales de EEG (sistema de procesamiento). Estas señales son altamente ruidosas y para capturar la información necesaria en ellas se utilizan técnicas de filtrado y de detección de patrones [1]. Los paradigmas son las instrucciones que sigue el usuario de la BCI y que permiten provocarle respuestas cerebrales conocidas que pueden ser detectadas por el sistema de procesamiento, y ser utilizadas para la actuación sobre el sistema externo, el cual puede ser un monitor o cualquier dispositivo controlado con señales digitales.

Según cual sea la aplicación a la que se destina el BCI, se utilizará el paradigma adecuado [3]-[5]. Uno de los más utilizados es la imagen motora (Motor Imagery) [6], consistente en que el sujeto se imagine el movimiento de una de sus manos durante unos segundos. Esta imaginación activa zonas de la corteza motora muy similares a las que se activan cuando se ejecuta el movimiento real de la mano. Así, las aplicaciones de BCI basadas en la imagen motora posibilitan la elección entre dos acciones en función de si uno se está imaginado el movimiento de una u otra mano.

Recientemente, los BCI basados en señales EEG y la imagen motora están comenzando a utilizarse en la neurorrehabilitación de pacientes que han perdido la movilidad en una de sus extremidades superiores como consecuencia de un ictus [7], [8]. También se están utilizando para la comunicación en pacientes con desorden de consciencia [9], [10]. 


\section{PROBLEMÁTICA}

Al no ser el cerebro una estructura idéntica entre diferentes personas, las señales EEG derivadas de los paradigmas pueden contener diferencias sutiles. Es por esto que en general los sistemas BCI precisan de una de calibración, o entrenamiento, previo a su uso, consiguiéndose así que el BCI se adapte a las especificaciones de las señales EEG de cada usuario.

Para conseguir esta adaptación se utiliza un clasificador de la señal de EEG. Debido a que la señal eléctrica del cerebro debe atravesar el cráneo y los tejidos de la cabeza, esta llega muy debilitada al amplificador y con una muy baja relación señal a ruido, y además con una baja resolución espacial. Para generar un clasificador bueno con todos estos impedimentos, se necesitan una cantidad alta de repeticiones de los patrones para poder promediarlos y obtener una relación señal a ruido superior. Cuantas más repeticiones se consigan, mejor calidad señal, y mejor clasificador.

En el caso de BCI basados en la imagen motora este proceso es más largo que en otros paradigmas, y en la mayoría de caso añade un tiempo importante e imprescindible para el control adecuado del sistema. En el caso de aplicaciones destinadas a la neurorrehabilitación donde los usuarios son pacientes con distintos grados de minusvalía, estos periodos largos de tiempo pueden llegar a ser una molestia. Y cuanto más corto sea el tiempo destinado a la calibración, mayor puede ser el tiempo destinado a la aplicabilidad del BCI, y mayor puede ser el beneficio conseguido.

\section{USO DE EMD PARA GENERAR NUEVAS TRAMAS}

\subsection{PARADIGMA DE IMAGEN MOTORA}

La imagen motora del sujeto se obtiene gracias a la definición de un paradigma. Este consiste en que el sujeto debe imaginarse haciendo la dorsiflexión de una de sus dos muñecas durante ocho segundos, durante los cuales se guardaran los datos de EEG. Cada segmento de ocho segundos del sujeto imaginándose el movimiento se llama trama (Figura 1). Al inicio de cada trama el sujeto recibirá una indicación sonora sobre cuál de las dos muñecas debe imaginarse el movimiento. El paradigma determinará de una forma pseudo-aleatoria el lado (derecho o izquierdo) en cada nueva trama, hasta obtener un número predeterminado de tramas iguales para cada lado.

Al finalizar el paradigma se tendrán un número $\mathrm{N}$ de tramas de señal EEG de 8 segundos para cada lado (N/2 para el lado derecho y N/2 para el lado izquierdo). Estos dos conjuntos de tramas son los utilizados para entrenar el clasificador que se utilizará en tramas posteriores para determinar el lado que el sujeto se está imaginando el movimiento.

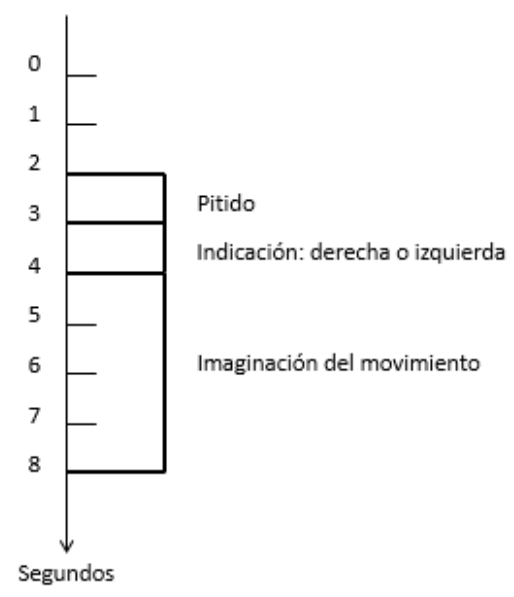

Figura 1: Trama del paradigma de imagen motora. Los primeros dos segundos el sujeto esta relajado. A los dos segundos suena un pitido y a continuación suena una indicación sonora del lado a imaginarse.

\subsection{GENERAR NUEVAS TRAMAS}

Como la calidad del clasificador es directamente proporcional al número de tramas de cada lado, tener un número elevado de tramas es vital para que el sujeto tenga un buen control del BCI [11]. Dadas las limitaciones para obtener un número elevado de tramas reales, se quiere estudiar la posibilidad de crear tramas artificiales a partir de las tramas reales ya existentes.

Por la naturaleza no lineal y no estacionaria de las señales EEG, se estima oportuno utilizar la descomposición EMD [12] para generar estas nuevas tramas artificiales.

\subsection{EL ALGORITMO EMD}

El algoritmo EMD (Empirical Mode Decomposition) es un método de descomposición de señales que permite analizar señales de naturaleza no-estacionaria y no-lineal.

La EMD descompone la señal en un número finito y pequeño de funciones intrínsecas llamadas IMFs (Interinsic Mode Functions) que representan las oscilaciones características de la señal. Cada IMF debe satisfacer dos condiciones: (i) el número de cruces por cero y el número de máximos será igual, o al menos diferente de uno, para todo el conjunto de datos; y (ii) en cualquier punto, el valor medio de la 
envolvente definida por el máximo local y la definida por el mínimo local, será cero [12].

Para una señal cualquiera, los pasos para obtener sus funciones IMF son los siguientes:

(i) determinar el máximo y el mínimo local para

(ii) generar la envolvente superior $\mathrm{e}$ inferior conectando esos máximos y mínimos locales respectivamente, con un método de interpolación

(iii) determinar la media local, haciendo la media de la envolvente superior e inferior de la señal.

(iv) sustraer la media local de los datos: a =

$$
-
$$

(v) si 0 cumple con el criterio de parada, se puede definir = o como a una IMF, sino se asigna = . y se vuelve a empezar el proceso en (i).

Una vez finalizado el algoritmo, la señal se pude definir a partir de sus IMFs (1). Quedando definida como:

$$
\mathrm{xt}=\operatorname{IMF} \mathrm{t}+\varepsilon \mathrm{t}
$$

Donde $n$ es el número de IMFs obtenidas de la EMD, y $\varepsilon$ t es una constante o la media de la tendencia.

\subsection{NUEVAS TRAMAS}

Como se puede apreciar en (1), una señal descompuesta con la EMD se puede recuperar completamente a partir de sus IMFs y del residuo. Del mismo modo, si cambiásemos una de sus IMFs por una IMF de otra señal descompuesta previamente, obtendríamos de (1) otra señal diferente. Con esta idea se pretende combinar diferentes IMFs de diferentes señales de EEG para crear una nueva señal EEG distinta a cualquiera de las señales originales que han contribuido con su IMF. Aun así, esta señal resultante de este proceso de creación artificial, mantendrá una estructura y unas características similares a las de las señales contribuidoras.

Una trama está formada por señales de EEG de ocho segundos de duración cada uno, teniendo tantas señales como canales EEG. Para crear nuevas tramas artificiales se utilizarán IMFs de otras tramas obtenidas de correr el paradigma. La selección de las tramas contribuidoras se hará aleatoriamente y solo se tendrá en cuenta que todas pertenezcan a la misma clase (lado derecho o lado izquierdo) que la trama artificial a generar, y que solo contribuían con una sola IMF para cada trama artificial nueva.

\section{MÉTODO}

\subsection{IMPLEMENTACIÓN DEL PARADIGMA}

La implementación del paradigma se ha realizado con un sistema (recoveriX de g.tec medical engineering $\mathrm{GmbH}$ ) que provee una interfaz gráfica que permite correr el paradigma y guardar los datos para su procesado posterior. El sistema está compuesto de un gorro EEG de 16 canales (g.SCARABEO de g.tec medical engineering $\mathrm{GmbH}$ ) puestos justo encima del córtex sensoriomotor según el sistema internacional 10/10: FC5, FC1, FCz, FC2, FC6, C5 C3, C1, Cz, C2, C4, C6, CP5, CP1, CP2, CP6. El electrodo de la posición Fpz está conectado a tierra, i en el lóbulo de la oreja derecha hay un electrodo de referencia. El gorro EEG está conectado a un amplificador biomédico (g.USBamp de g.tec medical engineering $\mathrm{GmbH})$, que a su vez está conectado mediante un cable USB al ordenador.

Cada vez que finaliza el paradigma el sistema proporciona los datos en bruto de donde se puede obtener las tramas para su procesado posterior, diferenciadas por el lado del movimiento a imaginar (derecha o izquierda). El paradigma recoge un total de 80 tramas: 40 para el lado derecho y 40 para el lado izquierdo. A este conjunto de tramas se le llama colección de tramas.

\subsection{PROCESADO DE LOS DATOS}

\subsubsection{Preprocesado de la señal}

Una vez obtenidos los datos estos se procesan en diferentes fases. Cada trama consta de 16 señales (una por canal) de 8 segundos con una frecuencia de muestreo de $256 \mathrm{~Hz}$, y una indicación de la clase a la que pertenece (derecha o izquierda). A cada una de las señales se les aplica un filtro digital de $8-30 \mathrm{~Hz}$.

\subsubsection{Obtención de un clasificador}

Para la obtención de un clasificador las tramas se separan en dos grupos según sea su clase (imaginación del movimiento de la muñeca derecho o izquierda), y a continuación se calcula un filtro espacial CSP (Common Spatial Pattern) [13][14].

Una vez obtenido los parámetros del CSP, utilizando solo los dos primeros y los dos últimos vectores, se aplican a todas las tramas, obteniéndose una matriz de datos para cada trama con 4 canales EEG de 8 segundos. A estas señales se efectúa un cálculo continuo de la varianza de su amplitud en una ventana de 1.5 segundos. Estos valores de varianza, una vez normalizados, representan las características que se utilizarán para entrenar el clasificador LDA (Linear Discriminant Analysis)[7]. 
Así, para un conjunto de tramas se obtiene un clasificador formado por los parámetros del CSP y los parámetros del LDA. Para asegurar que el clasificador no esté sobreentrenado se aplicará un proceso de validación cruzada.

\subsubsection{Cálculo de la tasa de error del clasificador}

Para la evaluación de un clasificador se utiliza su tasa de error. Una tasa de error elevada significa que el clasificador no es capaz de determinar a qué clase pertenecen las tramas. Para calcular la tasa de error se necesitan un clasificador y una colección de tramas (donde cada trama tendrá asociada una clase). A cada una de las tramas se las pasa por el clasificador, obteniendo a cada muestra (256 muestras cada segundo) y a la salida del clasificador el valor de la clase a la que pertenece. Si la clase resultante del clasificador no coincide con la real de la trama, esa muestra se indica como errónea. Una vez finalizadas todas las muestras de todas las trames se calcula el porcentaje de fallos, obteniendo la tasa de error.

La tasa de error se diferencia en dos valores: derecha e izquierda. De esta forma se puede diferenciar el error cuando se clasifican las tramas del lado derecho y las del lado izquierdo.

\subsection{CREACIÓN DE TRAMAS ARTIFICIALES}

A partir de una colección de tramas se creará una nueva colección de tramas en la cual se van a reemplazar un número determinado de tramas reales por tramas artificiales (el mismo número de tramas para cada una de las clases). Así, si se cambia una trama correspondiente al lado derecho, se debe también remplazar una del lado izquierdo.

Para proceder a hacer la transformación de la colección de tramas, se seleccionan aleatoriamente entre todas las tramas las que se van a reemplazar. Las otras tramas, serán las que contribuirán con sus IMFs en la obtención de las nuevas tramas artificiales. Para cada una de las tramas artificiales se seleccionará aleatoriamente cual es la trama que le cede su primera IMF, cual es la que les cede su segunda IMF, y así sucesivamente hasta obtener todas las IMFs. Con todas las IMFs seleccionadas aleatoriamente $\mathrm{y}$ aplicando la fórmula (1), obtenemos la trama artificial.

Como una trama está compuesta de 16 canales, cada trama que cede su IMF para una trama nueva, en realidad cederá las 16 IMF que corresponden a sus 16 canales. Así, en realidad se están creando 16 señales nuevas para cada trama.

\subsection{PROCEDIMIENTO}

Para el experimento solo se utiliza una única colección de 80 tramas (40 para cada clase) que se han obtenido de un sujeto sano de 37 años. A partir de esta colección de tramas se crean otras ocho colecciones de tramas, cada una con un número diferente de tramas artificiales: 2, 4, 6, 8, 10, 20, 30 y 40. Para cada colección de tramas se calcula su clasificador, obteniendo un total de nueve clasificadores (el de la colección original y los ocho de las colecciones con tramas artificiales).

El siguiente paso es calcular la tasa de error de cada uno de los nueve clasificadores, utilizando los datos de la colección de tramas originales. No se van a considerar todas las muestras de los ocho segundos que dura una trama, sino que solo se consideran las muestres a partir de los 3.5 segundos hasta el final de la trama. Es en estos momentos cuando el usuario ya ha recibido la señal acústica y ya se está imaginando el movimiento de la muñeca.

\section{RESULTADOS}

En la Tabla 1 se muestran los resultados del experimento para cada uno de los clasificadores y para cada una de las clases.

Tabla 1: Tasa de error de cada clasificador

\begin{tabular}{|l|c|c|}
\hline \multicolumn{1}{|c|}{ Clasificador } & Derecha & Izquierda \\
\hline 0 tramas artificiales & 6,63 & 2,66 \\
\hline 2 tramas artificiales & 8,30 & 3,21 \\
\hline 4 tramas artificiales & 7,66 & 2,26 \\
\hline 6 tramas artificiales & 9,05 & 3,38 \\
\hline 8 tramas artificiales & 9,23 & 1,36 \\
\hline 10 tramas artificiales & 8,47 & 1,80 \\
\hline 20 tramas artificiales & 11,51 & 0,92 \\
\hline 30 tramas artificiales & 6,72 & 4,05 \\
\hline 40 tramas artificiales & 3,62 & 6,62 \\
\hline
\end{tabular}

El primer clasificador es el que se ha generado con la colección de tramas original, y comparando sus resultados con los de los otros clasificadores se puede ver que el comportamiento es siempre mejor para el lado izquierdo que para el derecho, exceptuando los dos últimos clasificadores. También se puede apreciar que cuantas más tramas artificiales en la colección, mejor es la tasa de error para el lado izquierdo y peor para el lado derecho, exceptuando también los dos últimos casos. 
Los dos últimos clasificadores tienen unas tasas de errores bajas y muy similares a las del clasificador sin tramar artificiales, a pesar de tener un porcentaje muy elevado de tramas artificiales en la colección que los generó.

\section{DISCUSIÓN}

Los resultados del experimento muestran que todos los clasificadores tienen una tasa de error similar a la tasa de error del clasificador generado con la colección de tramas original. Esto quiere decir que se podría utilizar uno de los clasificadores con tramas artificiales para utilizar el BCI, siendo el proceso de entrenamiento más corto porqué se hubieran precisado menos tramas reales para obtener la colección de tramas.

Estos buenos resultados se pueden explicar por cuatro motivos:

(i) La descomposición EMD se adapta muy bien a señales no-lineales y no-estacionarias como es el caso de las señales EEG.

(ii) Usando las IMFs de tramas reales se recoge su información subyacente que estará en las tramas artificiales.

(iii) Los clasificadores LDA tienen poca tendencia al sobreentrenamiento y una gran capacidad de generalización.

(iv) El clasificador LDA se obtiene después de una validación cruzada que ayuda a prevenir el sobreentrenamiento.

A pesar de todo esto, los resultados solo son preliminares, y es necesario validarlos estadísticamente para asegurar que es posible utilizar tramas artificiales de la colección existente para aumentar el número total de tramas. Para la validación estadística son imprescindibles la repetición varias veces del mismo experimento utilizando diferentes IMFs de diferentes tramas para crear las tramas artificiales. También será imprescindible repetir el experimento con un número suficiente de sujetos diferentes.

\section{Agradecimientos}

Programa de Doctorados Industriales, dentro del Departamento de Empresa y Conocimiento de la Generalitat de Catalunya.

\section{Referencias}

[1] J. R. Wolpaw, N. Birbaumer, D. J. McFarland, G. Pfurtscheller, and T. M. Vaughan, "Brain
Computer Interfaces for communication and control," Front. Neurosci., vol. 4, no. 113, pp. 767-791, 2002.

[2] J. J. Shih, D. J. Krusienski, and J. R. Wolpaw, "Brain-computer interfaces in medicine.," Mayo Clin. Proc., vol. 87, no. 3, pp. 268-79, Mar. 2012.

[3] M. Oehler, P. Neumann, M. Becker, G. Curio, and M. Schilling, "Extraction of SSVEP signals of a capacitive EEG helmet for human machine interface.," Conf. Proc. IEEE Eng. Med. Biol. Soc., vol. 2008, no. factor 2, pp. 4495-4498, 2008.

[4] G. Pfurtscheller, "Functional brain imaging based on ERD/ERS," Vision Res., vol. 41, no. 10-11, pp. 1257-1260, May 2001.

[5] L. A. Farwell and E. Donchin, "Talking off the top of your head: toward a mental prosthesis utilizing event-related brain potentials.," Electroencephalogr. Clin. Neurophysiol., vol. 70, no. 6, pp. 510-23, Dec. 1988.

[6] C. Guger, C. Kapeller, R. Ortner, and K. Kamada, "Motor Imagery with BrainComputer Interface Neurotechnology,” 2015.

[7] W. Cho et al., "Paired Associative Stimulation Using Brain-Computer Interfaces for Stroke Rehabilitation: A Pilot Study.," Eur. J. Transl. Myol., vol. 26, no. 3, p. 6132, 2016.

[8] A. Ramos-Murguialday et al., "Brainmachine interface in chronic stroke rehabilitation: a controlled study.," Ann. Neurol., vol. 74, no. 1, pp. 100-8, Jul. 2013.

[9] C. Guger et al., "Complete Locked-in and Locked-in patients: Command following assessment and communication with vibrotactile P300 and motor imagery braincomputer interface tools," Front. Neurosci., vol. 11, no. May, p. 251, 2017.

[10] C. Guger et al., Brain-Computer Interfaces for Assessment and Communication in Disorders of Consciousness, no. January. 2013.

[11] H. Ramoser, J. Muller-Gerking, and G. Pfurtscheller, "Optimal spatial filtering of single trial EEG during imagined hand 
movement," IEEE Trans. Rehabil. Eng., vol. 8, no. 4, pp. 441-446, Dec. 2000.

[12] N. Huang et al., "The empirical mode decomposition and the Hilbert spectrum for nonlinear and non-stationary time series analysis," Proc. R. Soc. A Math. Phys. Eng. Sci., vol. 454, no. 1971, pp. 995, 903, 1998.

[13] Z. J. Koles, M. S. Lazar, and S. Z. Zhou, "Spatial patterns underlying population differences in the background EEG," Brain Topogr., vol. 2, no. 4, pp. 275-284, 1990.

[14] Yijun Wang, Shangkai Gao, and Xiaornog Gao, "Common Spatial Pattern Method for Channel Selelction in Motor Imagery Based Brain-computer Interface," in 2005 IEEE Engineering in Medicine and Biology 27th Annual Conference, 2005, vol. 5, pp. 53925395. 